\title{
Increased Levels of Oxidative Damage in Liver Metastases Compared with Corresponding Primary Colorectal Tumors
}

\section{Association with Molecular Subtype and Prior Treatment}

\author{
Lizet M. van der Waals, ${ }^{*}$ Jennifer M.J. Jongen, ${ }^{*}$ Sjoerd G. Elias, ${ }^{\dagger}$ Kateryna Veremiyenko, ${ }^{*}$ Kari Trumpi, ${ }^{*}$ Anne Trinh, ${ }^{\ddagger}$ \\ Jamila Laoukili, ${ }^{*}$ Inge Ubink, ${ }^{*}$ Susanne J. Schenning-van Schelven, ${ }^{*}$ Paul J. van Diest, ${ }^{\S}$ Inne H.M. Borel Rinkes, ${ }^{*}$ and \\ Onno Kranenburg*
}

From the Department of Surgical Oncology, * UMC Utrecht Cancer Center, the Julius Center for Health Sciences and Primary Care, ${ }^{\dagger}$ and the Department of Pathology, ${ }^{\S}$ University Medical Center Utrecht, Utrecht University, Utrecht, the Netherlands; and the Department of Medical Oncology, ${ }^{\ddagger}$ Dana-Farber Cancer Institute, Boston, Massachusetts

\author{
Accepted for publication \\ June 19, 2018. \\ Address correspondence to \\ Onno Kranenburg, Ph.D., \\ Division of Biomedical \\ Genetics, University Medical \\ Center Utrecht, PO Box 85500 \\ 3508 GA Utrecht, the Nether- \\ lands. E-mail: o.kranenburg@ \\ umcutrecht.nl.
}

\begin{abstract}
High levels of oxidative stress in disseminated colorectal cancer tumor cells may form a therapeutically exploitable vulnerability. However, it is unclear whether oxidative stress and damage persist in metastases. Therefore, we analyzed markers of oxidative damage in primary colorectal tumors and their corresponding liver metastases. Markers of generic and oxidative DNA damage [phosphorylated histone $\mathrm{H} 2 \mathrm{AX}(\gamma \mathrm{H} 2 \mathrm{AX})$ and 8-hydroxy-2'-deoxyguanosine (8-0HdG)] were significantly higher in liver metastases compared with their corresponding primary tumors. Chemotherapy and/or radiotherapy before tumor resection was associated with increased persistent oxidative DNA damage, and this effect was more pronounced in metastases. Immunohistochemistry-based molecular classification into epithelialand mesenchymal-like molecular subtypes revealed that untreated mesenchymal-like tumors contained lower levels of oxidative DNA damage compared with epithelial-like tumors. Treated mesenchymal-like tumors, but not epithelial-like tumors, showed significantly higher levels of $\gamma \mathrm{H} 2 \mathrm{AX}$ and 8-0HdG. Mesenchymal-like tumors expressed significantly lower levels of phosphorylated nuclear factor erythroid 2-related factor 2, a master regulator of the antioxidant response, and nuclear factor erythroid 2-related factor 2-controlled genes. Of interest, a positive $8-0 \mathrm{HdG}$ status identified a subgroup of mesenchymallike metastases with a poor overall survival. An increased capacity to tolerate therapy-induced oxidative damage in mesenchymal-like colorectal cancer may explain, at least in part, the poor responsiveness of these tumors to chemotherapy, which could contribute to the poor survival of this patient subgroup. (Am J Pathol 2018, 188: 2369-2377; https://doi.org/10.1016/j.ajpath.2018.06.008)
\end{abstract}

Colorectal cancer (CRC) is frequently a lethal disease. ${ }^{1,2}$ Approximately $20 \%$ of patients with CRC present with metastases at diagnosis. ${ }^{2}$ Despite multiple improvements in cancer care, such as more effective systemic therapies and improved surgical techniques, the median overall survival (OS) of metastatic CRC (mCRC) after chemotherapy combined with targeted agents is still poor, up to approximately 30 months. ${ }^{3,4}$ Clearly, more effective treatment approaches are needed. Characterization of the dependencies and
Supported by the Gieskes-Strijbis Foundation (L.M.W.); Dutch Cancer Society grants UU2013-5865 (J.M.J.J.), 2015-8088 (J.L. and S.J.S.), and 2014-6617 (I.U.); and Vrienden van het UMC Utrecht (K.T.).

Disclosures: None declared.

Current address of J.M.J.J., Department of Surgery, Meander Medical Center, Amersfoort, the Netherlands; of K.T., Department of Surgery, Diakonessenhuis, Utrecht, the Netherlands. 
vulnerabilities of metastasized tumor cells may help in designing such therapies.

Oxidative stress is characterized by an imbalance between the production of reactive oxygen species (ROS) and their neutralization by antioxidants. Oxidative stress is known to be a common characteristic of cancer cells that can be exploited therapeutically. ${ }^{5,6}$ Moderately increased levels of ROS stimulate tumorigenesis by acting as second messengers in tumor-promoting signaling pathways. ${ }^{7}$ Antioxidants are important for maintaining the reduction-oxidation (redox) balance in tumor cells, thereby preventing cell death and senescence. ${ }^{6}$ Of interest, metastasizing tumor cells experience high levels of oxidative stress, which is an important barrier to successful establishment of distant metastases. $^{8-10}$ However, not much is known about oxidative stress in established metastases, which are the main targets of systemic therapy in mCRC. Chemotherapy regimens for mCRC are based on fluoropyrimidines (5-fluorouracil or capecitabine) in combination with oxaliplatin and/or irinotecan. ${ }^{4}$ These chemotherapeutic drugs work, at least in part, by enhancing ROS levels and causing oxidative damage. ${ }^{11,12}$ Moreover, a signature reflecting increased oxidative signaling has been associated with the aggressive mesenchymal (MES) CRC subtype, now commonly referred to as consensus molecular subtype 4 (CMS4). ${ }^{13}$

Increased oxidative stress during metastatic outgrowth, after chemotherapy or as a consequence of aggressive tumor biology, could generate a therapeutically exploitable vulnerability. ${ }^{14}$ To gain insight into patient subgroup(s) that might benefit from such a therapeutic approach, we assessed the levels of oxidative damage and expression of an antioxidant transcription factor in a large series of primary CRC and corresponding colorectal liver metastases (CRLMs) in relation to molecular subtypes. During data analysis, prior chemotherapy and/or radiotherapy and molecular subtype were considered as potential factors influencing oxidative stress status.
A
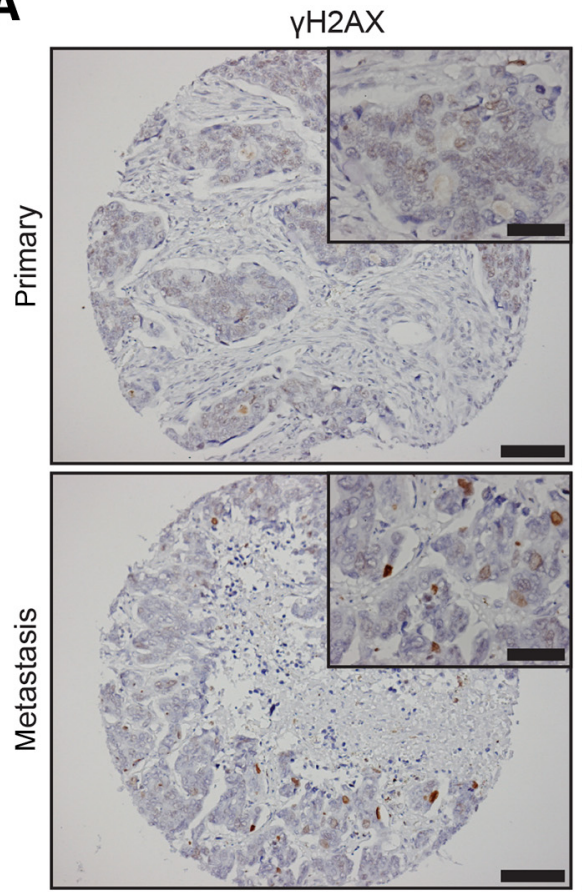

B

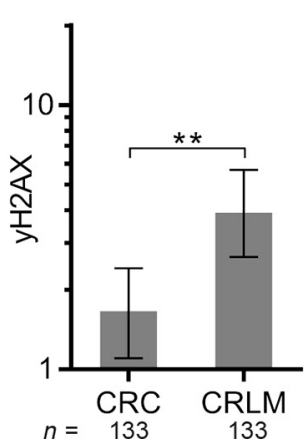

8-OHdG
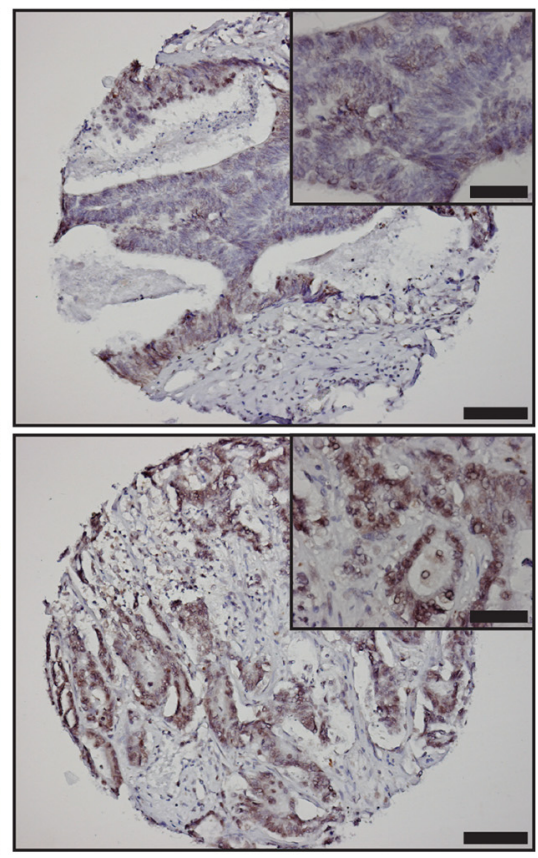

C

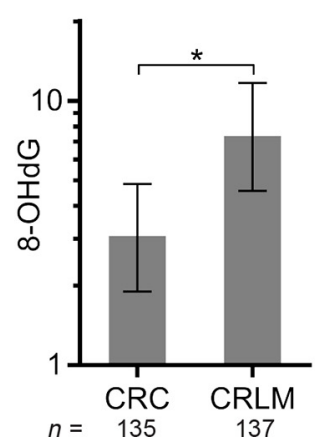

Figure 1 Increased oxidative DNA damage in colorectal liver metastases. A: Representative images of immunohistochemical expression of phosphorylated histone $\mathrm{H} 2 \mathrm{AX} \quad(\gamma \mathrm{H} 2 \mathrm{AX})$ and 8-hydroxy-2'-deoxyguanosine (8-OHdG) in primary colorectal cancer (CRC) samples and corresponding colorectal liver metastases (CRLMs). Images of $10 \times$ microscope objective whole-tissue microarray tissue cores and $40 \times$ microscope objective (insets) are shown. B and C: Bar graphs showing quantification of $\gamma \mathrm{H} 2 \mathrm{AX}$ expression (B) and 8OHdG accumulation (C) in CRC tumors versus CRLMs. B and C: A paired $t$-test was applied to the log-transformed data to compare $\gamma \mathrm{H} 2 \mathrm{AX}$ expression and $8-0 \mathrm{HdG}$ accumulation as continuous variable in CRC versus CRLMs. Error bars represent the geometric means and corresponding $95 \%$ CIs (B and C). ${ }^{*} P \leq 0.05,{ }^{*} * P \leq 0.01$. Scale bars: 100 $\mu \mathrm{m}$ (A, main images); $50 \mu \mathrm{m}$ (A, insets). 


\section{Materials and Methods}

\section{Patients and Tissues}

The patient cohort was described before. ${ }^{15}$ CRC patients who underwent a resection for CRLMs at the University Medical Center Utrecht (Utrecht, the Netherlands) between 2003 and 2014 were included in a prospective database. Their primary tumors were resected in the University Medical Center Utrecht, Diakonessenhuis Utrecht, St. Antonius Hospital Nieuwegein, or Rijnstate Hospital at Arnhem. Patients with either synchronous or metachronous CRLMs were included. Eventually, 158 mCRC patients were included. Clinicopathological data were available from 129 patients ( 86 males and 43 females). Median follow-up time was 76 months. Mean age was 62 years (range, 37 to 83 years) at the time of surgery for liver metastases. The use of anonymous or coded leftover material for scientific purposes is exempt from informed consent, according to Dutch law. Standard treatment agreements include an opt-out for such use. ${ }^{16,17}$

\section{TMA Construction}

A 0.6-mm (triple-core) tissue microarray (TMA) of primary CRC tumors and corresponding CRLMs was constructed, ${ }^{15}$ from which multiple sections ( $4 \mu \mathrm{m}$ thick) were cut for immunohistochemical staining.

\section{Treatment Status}

Primary tumors were considered treated when neoadjuvant chemotherapy and/or radiotherapy was administered. Tissue from liver metastases was considered treated when patients received neoadjuvant chemotherapy for metachronous CRLMs. Tissue from synchronous metastases was considered treated when neoadjuvant chemotherapy for primary tumors and/or liver metastases was administered. An overview of treatment status is given in Supplemental Table S1.

\section{Mesenchymal versus Epithelial Subtypes}

All tumors included within the TMA were previously classified as MES-like or epithelial (EPI)-like, ${ }^{15}$ using a validated immunohistochemistry-based classifier. ${ }^{18}$ In short, this assay identifies EPI- and MES-like CRC tumors using expression of homeobox protein CDX-2 (CDX2), FERM domain-containing protein 6 (FRMD6), 5-hydroxytryptamine receptor 2B (HTR2B), zinc finger E-box-binding homeobox 1 (ZEB1), and pan-cytokeratin. High expression of the differentiation marker CDX2 and low immunohistochemical expression of the epithelial-mesenchymal marker ZEB1 identify EPI-like cancers and increased levels of the vasoactive neurotransmitter HTR2B and goblet cell marker FRMD6 MES-like cancers. In addition, pan-cytokeratin was used to normalize the other markers for epithelial content.
On the basis of the immunohistochemistry staining per marker, a prediction probability for the MES-like subtype was calculated (MES probability score). Each individual TMA core was classified as EPI- or MES-like using an MES probability cutoff of 0.6 (EPI $<0.6$; MES $\geq 0.6$ ), and subtype classification was obtained by majority consensus scoring. This individual per core MES probability score was used to look for putative correlations between per core marker expression and the MES probability scoring.

\section{Immunohistochemistry}

Three immunohistochemical stainings were performed on serial sections of the TMA: phosphorylated histone H2AX $(\gamma \mathrm{H} 2 \mathrm{AX})$, 8-hydroxy-2'-deoxyguanosine (8-OHdG), and phosphorylated nuclear factor erythroid 2-related factor 2 (NRF2; alias NFE2L2). Supplemental Table S2 gives an overview of antibodies and dilutions, incubation conditions, and antigen-retrieval methods for each marker. For all stainings, sections were deparaffinized with xylene and rehydrated with serial dilutions of ethanol and water. Next, endogenous peroxidase activity was blocked with $1.5 \%$ hydrogen peroxide diluted in phosphate-buffered saline for
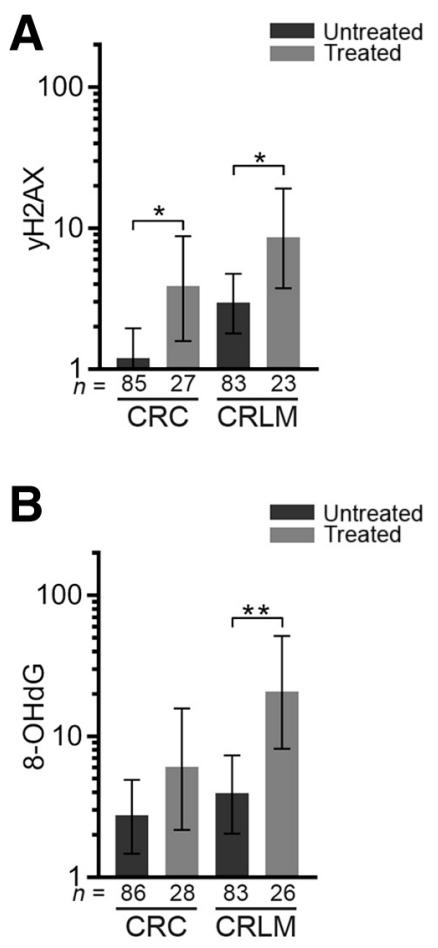

Figure 2 Prior treatment is associated with DNA damage and 8-0HdG accumulation. $\mathbf{A}$ and $\mathbf{B}$ : Bar graphs showing quantification of phosphorylated histone $\mathrm{H} 2 \mathrm{AX}(\gamma \mathrm{H} 2 \mathrm{AX})$ expression $(\mathbf{A})$ or 8-hydroxy-2'-deoxyguanosine $(8-0 \mathrm{HdG})$ accumulation (B) in primary colorectal cancer (CRC) and colorectal liver metastases (CRLMs), treated versus untreated tumors. A: Therapy-exposed CRC and CRLMs express higher levels of the DNA damage marker $\gamma \mathrm{H} 2 \mathrm{AX}$. B: Therapy-exposed CRC and CRLMs accumulate 8-OHdG. A and B: An unpaired $t$-test was applied to the log-transformed data to compare groups. Error bars represent the geometric means and corresponding $95 \%$ CIs (A and $\mathbf{B})$. ${ }^{*} P \leq 0.05,{ }^{*} P \leq 0.01$. 
30 minutes. Then, antigen retrieval was performed by boiling samples for 20 minutes in citrate buffer $(\mathrm{pH}$ 6.0) for $\gamma \mathrm{H} 2 \mathrm{AX}$ and 8-OHdG or EDTA buffer (pH 9.0) for phosphorylated NRF2. Protein Block (Novolink Polymer Detection Systems, Leica Biosystems, Newcastle upon Tyne, UK) was applied, according to the manufacturer's instructions, to block nonspecific binding of the anti-8OHdG antibody. Primary antibody incubation was performed as follows: anti- $\gamma \mathrm{H} 2 \mathrm{AX}$ (Ser139; rabbit; Cell Signaling, Danvers, MA; 2577; 1:50; overnight at $4^{\circ} \mathrm{C}$ ), anti-8-OHdG (N45.1; mouse monoclonal; Abcam, Cambridge, UK; ab48508; 1:500; 1 hour at room temperature), and anti-NRF2 (phosphorylated S40; rabbit monoclonal; Abcam; ab76026; 1:500; 1 hour at room temperature). Subsequently, the secondary antibody BrightVision poly-horseradish peroxidase-conjugated anti-rabbit or anti-mouse IgG (ImmunoLogic, Duiven, the Netherlands) was incubated for 30 minutes at room temperature. All sections were developed with diaminobenzidine for $10 \mathrm{mi}-$ nutes, followed by a hematoxylin counterstaining. Air-dried slides were mounted in a ClearVue Coverslipper (Thermo Fisher Scientific, Waltham, MA). To check for nonspecific binding of the secondary antibody, slides were incubated without primary antibodies in the presence of the secondary antibody. Tissues previously validated in the literature as positive controls were used throughout.

\section{Evaluation of Staining}

Scoring was performed by consensus of two investigators (L.M.W. and K.V.) blinded to clinicopathological data and
EPI- versus MES-like subtype, after training by a pathologist (P.J.D.). Damaged and empty TMA cores and those not containing cancer cells were excluded. For $8-\mathrm{OHdG}$ and phosphorylated NRF2, nuclear staining intensity was scored as 0 (negative), 1 (weak), 2 (moderate), or 3 (strong) (Supplemental Figure S1), ignoring cytoplasmic staining or staining within clearly necrotic regions. For $8-\mathrm{OHdG}$ and phosphorylated NRF2, the percentage of positive nuclei was scored per intensity, calculating $\mathrm{H}$-scores ${ }^{19}$ as follows:

$[1 \times(\%$ cells with intensity 1$)+2 \times(\%$ cells with intensity 2$)+3 \times(\%$ cells with intensity 3$)]$.

Because of the highly heterogeneous intensity of the nuclear $\gamma \mathrm{H} 2 \mathrm{AX}$ staining per cell, only the percentage of positive tumor cells was scored. The mean $\mathrm{H}$-scores $(8-\mathrm{OHdG}$ and phosphorylated NRF2) or percentage positivity $(\gamma \mathrm{H} 2 \mathrm{AX})$ of three cores for each primary tumor specimen or metastatic specimen per patient were calculated. TMA raw data are provided in Supplemental Table S3.

\section{Bioinformatic Analysis}

All bioinformatic analyses were performed using the R2 Genomics Analysis and Visualization Platform (https:// hgserver1.amc.nl/cgi-bin/r2/main.cgi, registration required). Expression data were obtained from a large composite primary CRC cohort data set and a CRLM cohort data set. ${ }^{13,20}$

\section{Statistical Analysis}

All analyses were performed using IBM SPSS statistics version 23 (IBM Corp., Armonk, NY) and Excel version
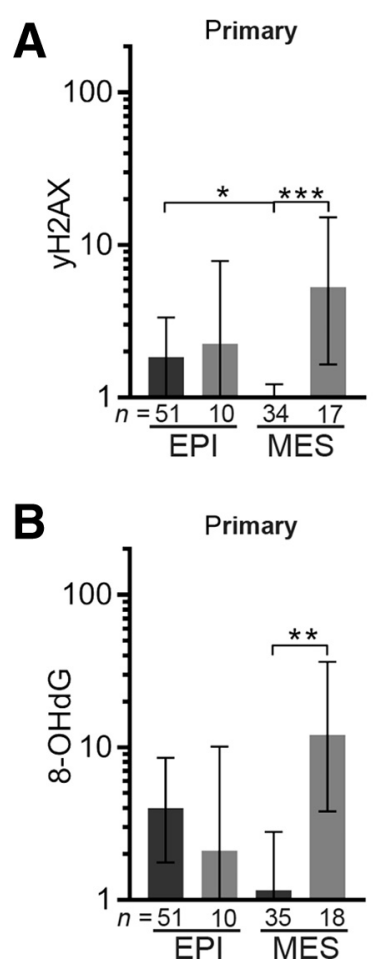
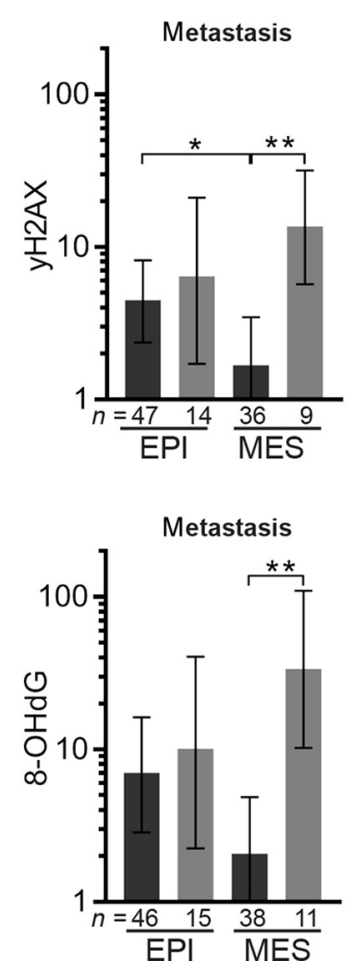

Figure 3 0xidative DNA damage is increased in treatmentexposed mesenchymal (MES)-like tumors characterized by low basal levels of DNA damage. A: Bar graphs showing expression of phosphorylated histone $\mathrm{H} 2 \mathrm{AX}(\gamma \mathrm{H} 2 \mathrm{AX})$ in primary colorectal cancer and colorectal liver metastases stratified according to treatment status and subtype. B: Bar graphs showing levels of 8-hydroxy-2'deoxyguanosine $(8-\mathrm{OHdG})$ in primary colorectal cancer and colorectal liver metastases stratified according to treatment status and subtype. A and B: A one-way analysis of variance and Fisher's least significant difference post hoc test was applied to the logtransformed data to compare groups. Error bars represent the geometric means and corresponding $95 \%$ CIs (A and $\mathbf{B}) .{ }^{*} P \leq 0.05$, $* * P \leq 0.01$, and ${ }^{* * *} P \leq 0.001$. EPI, epithelial. 
A
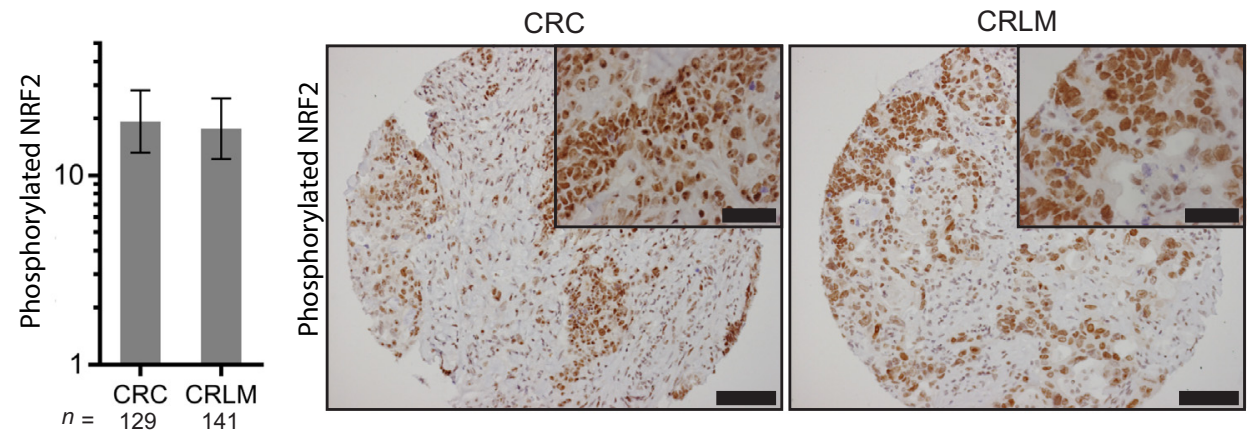

B
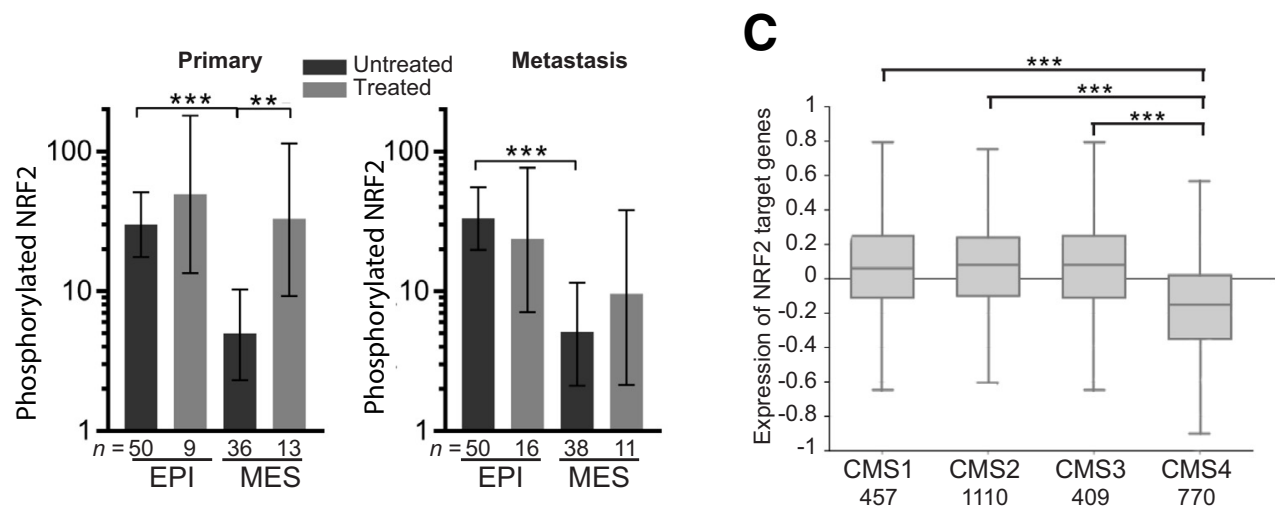

D
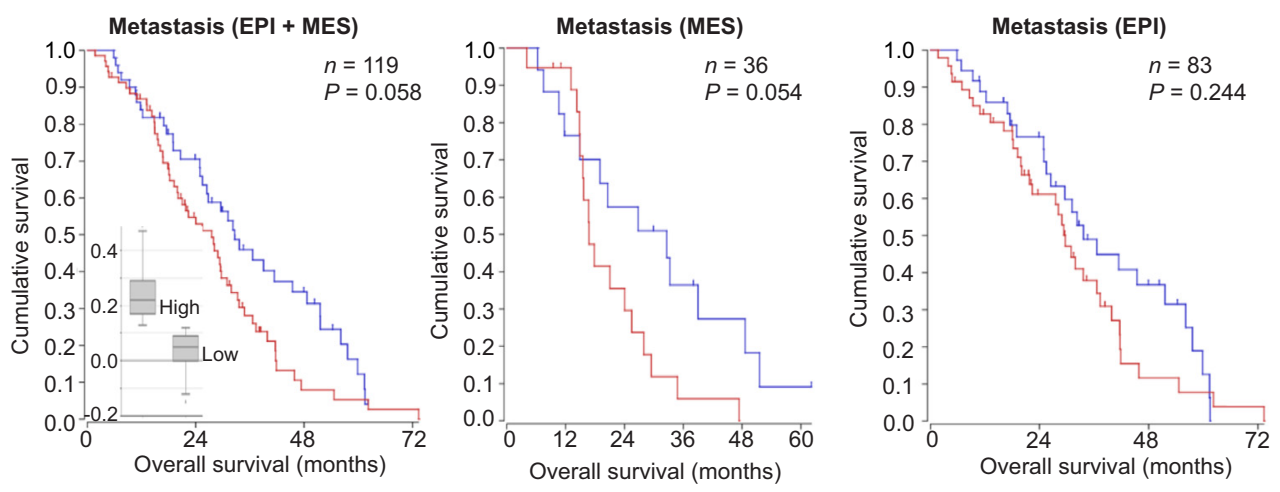

$\begin{array}{ll}\neg \text { High NRF2 } & \text { + High NRF2-censored } \\ \square \text { Low NRF2 } & \text { । Low NRF2-censored }\end{array}$

Figure 4 Low basal levels of phosphorylated nuclear factor erythroid 2-related factor 2 (NRF2) and its target genes in mesenchymal (MES)-like tumors. A: Bar graph showing quantification of phosphorylated NRF2 expression and representative images of immunohistochemical nuclear expression of phosphorylated NRF2 in primary colorectal cancer (CRC) and corresponding colorectal liver metastases (CRLMs). Images of 10× microscope objective whole-tissue microarray tissue cores and $40 \times$ microscope objective (insets) are shown. A paired $t$-test was applied to the log-transformed data to compare phosphorylated NRF2 expression as a continuous variable in CRC versus CRLMs. B: Low basal levels of phosphorylated NRF2 in mesenchymal-like tumors. A one-way analysis of variance and Fisher's least significant difference post hoc test were applied to the log-transformed data to compare groups. C: Expression of NRF2 target genes within 3232 colorectal cancer tumors classified according to their molecular subtype. ${ }^{13}$ The following NRF2 target genes were included in the analyses: $A B C C 4$, ALDH3A1, AOX1, CAT, CHAC1, ENTPD5, FAM3B, GCLC, GCLM, GSR, HMOX1, HTATIP2, IDH1, LRP8, MAGOHB, MGST1, MOCOS, NQ01, PRR13, RNF183, SLC19A2, SLC22A23, SLC7A11, TRIB3, VASN, and ZFP36. Analysis of variance $P$ values were determined. The box represents the median and middle $50 \%$ of the scores. The upper and lower whiskers represent scores outside the middle 50\%. D: Kaplan-Meier curves showing the differences in overall survival between NRF2 high and low subgroups in a cohort consisting of patients undergoing resection for their colorectal liver metastases. ${ }^{20}$ Levels of NRF2 mRNA in liver metastases were used to generate high and low subgroups, using the average as a cutoff point. NRF2 mRNA levels in both groups are shown in the inset. Significance was tested using the log-rank test. Error bars represent the geometric means and corresponding $95 \%$ CIs (A and $\mathbf{B}) .{ }^{* *} P \leq 0.01,{ }^{* * *} P \leq 0.001$. Scale bars: $100 \mu \mathrm{m}$ (A, main images); $50 \mu \mathrm{m}$ (A, insets). CMS, consensus molecular subtype; EPI, epithelial.

2013 (Microsoft Corp., Redmond, WA). GraphPad Prism version 7.0 for Windows (GraphPad Software, La Jolla, CA) was used for graphical presentation of the data. $P<0.05$ (two tailed) was considered statistically significant. All clinicopathological characteristics of the patients were assessed using a $\chi^{2}$ test and an independent-sample $t$-test for the continuous variable age. Correlations between expression of the different immunohistochemical markers within primary CRC tissue or CRLM tissue cores and MES probability scoring 
Table 1 Correlations between Expression of the Different Immunohistochemical Markers within Primary CRC Tissue or CRLM Tissue Cores and Mesenchymal Probability Scoring

\begin{tabular}{|c|c|c|c|c|c|c|c|c|}
\hline \multirow[b]{2}{*}{ Marker } & \multicolumn{4}{|l|}{ CRC } & \multicolumn{4}{|c|}{ CRLM tissue core } \\
\hline & Kendall's $\tau^{*}$ & $95 \% \mathrm{CI}$ & $P$ value $^{\dagger}$ & $n^{\ddagger}$ & Kendall's $\tau^{*}$ & $95 \% \mathrm{CI}$ & $P$ value $^{\dagger}$ & $n^{\ddagger}$ \\
\hline$\gamma \mathrm{H} 2 \mathrm{AX}$ & -0.130 & -0.263 to 0.002 & 0.0540 & 220 & -0.057 & -0.190 to 0.075 & 0.398 & 201 \\
\hline Phosphorylated NRF2 & -0.206 & -0.312 to -0.099 & 0.0002 & 220 & -0.142 & -0.272 to -0.012 & 0.0318 & 214 \\
\hline
\end{tabular}

*Kendall's rank correlation coefficient (Kendall's $\tau$ ).

${ }^{\dagger}$ Two-sided $P$ values are given.

${ }^{\ddagger} n=$ number of $\mathrm{XY}$ pairs.

8-OHdG, 8-hydroxy-2'-deoxyguanosine; CRC, colorectal cancer; CRLM, colorectal liver metastasis; $\gamma H 2 A X$, phosphorylated histone H2AX; NRF2, nuclear factor erythroid 2-related factor 2.

were determined using the Kendall's $\tau$ test. Hydrogen peroxide metagene expression within CRC molecular subtypes was compared using a one-way analysis of variance. Because the immunohistochemistry expression data were right skewed, the data were log transformed before analyzing and comparing mean differences in expression. The results are reported on the original scale by back transformation, yielding geometric means and corresponding 95\% CIs. To account for values of zero using this approach, 0.5 was added to all data points before log transformation, and this value was subtracted again from the back-transformed results. A paired $t$-test was applied to the log-transformed data to compare $\gamma \mathrm{H} 2 \mathrm{AX}$ expression, 8-OHdG accumulation, and phosphorylated NRF2 expression in primary CRC samples and corresponding CRLMs; an independent-sample $t$-test was used to compare expression in treated and untreated tumor samples. All other outcomes were compared using a one-way analysis of variance, followed by a Fisher's least significant difference test, to compare individual groups. For survival analysis, OS was measured from time of liver surgery until death or end of follow-up. Kaplan-Meier survival analyses were performed, and survival curves were compared between the different groups by log-rank tests.

\section{Results}

Increased 0xidative DNA Damage in Liver Metastases Compared with Primary Colorectal Tumors

To study DNA damage accumulation in primary CRC tumors and corresponding liver metastases, phosphorylation of histone $\mathrm{H} 2 \mathrm{AX}$ was analyzed as a marker of the formation of DNA double-strand breaks. $\gamma \mathrm{H} 2 \mathrm{AX}$ initiates repair of double-strand breaks and is a useful marker for their quantification. $^{21} \quad \gamma \mathrm{H} 2 \mathrm{AX}$ levels were significantly increased $(P \leq 0.01)$ in liver metastases compared with corresponding primary tumors (Figure 1, A and B), indicating an increased load of DNA double-strand breaks or repair in mCRC. ROS may cause oxidative damage to the nucleotide precursor pool, which-on incorporation into DNA - can lead to DNA damage. DNA bases, predominantly guanine, are also susceptible to direct oxidation by ROS, and the oxidized base $8-\mathrm{OHdG}$ is, therefore, a frequently used marker of oxidative DNA damage. ${ }^{22} \mathrm{~A}$ significantly increased accumulation of 8 -OHdG $(P \leq 0.05)$ was observed in metastases compared with the primary tumors from which they were derived (Figure 1, A and C).
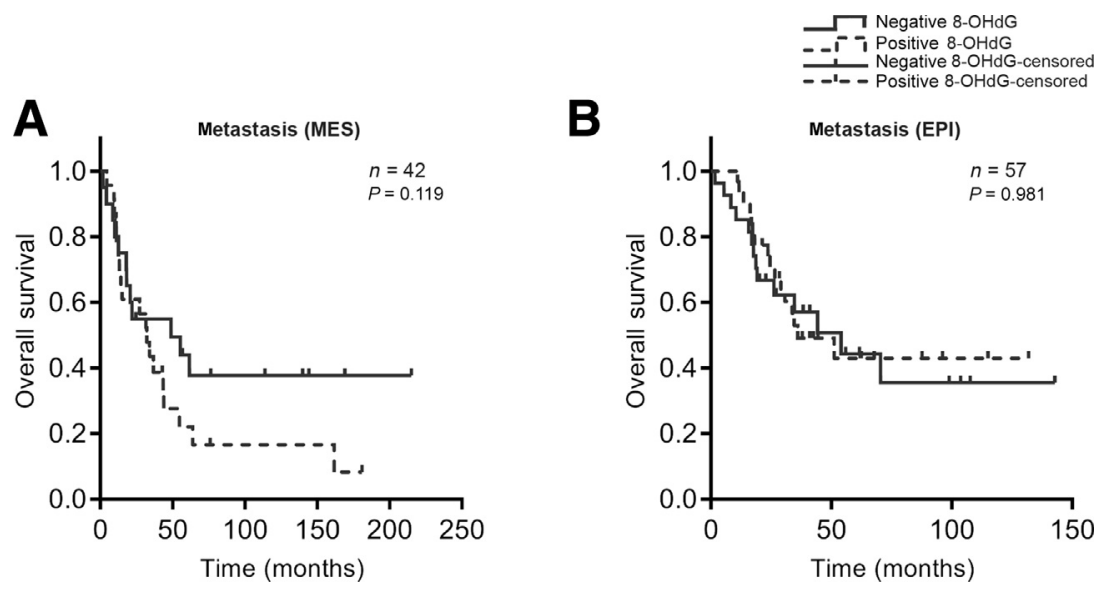

Figure 5 8-hydroxy-2'-deoxyguanosine (8-OHdG) positivity identifies a group of poor-prognostic mesenchymal (MES)-like metastases. A: Kaplan-Meier curves showing the differences in overall survival between $8-0 \mathrm{HdG}$-positive and $8-0 \mathrm{HdG}$-negative MESlike metastases. Significance was tested using the logrank test. B: Kaplan-Meier curves showing the differences in overall survival between $8-0 \mathrm{HdG}$-positive and 8-OHdG-negative epithelial (EPI)-like metastases. Significance was tested using the log-rank test. 
Table 2 Patient and Liver Metastasis Characteristics of the Classified Patients

\begin{tabular}{|c|c|c|c|c|c|c|c|c|c|c|c|c|c|c|}
\hline \multirow[b]{3}{*}{ Characteristics } & \multicolumn{7}{|c|}{ Epithelial-like metastases $(n=69)$} & \multicolumn{7}{|c|}{ Mesenchymal-like metastases $(n=60)$} \\
\hline & \multicolumn{2}{|c|}{$\begin{array}{l}8-0 \mathrm{HdG} \\
\text { UNK } \\
(n=11) \\
\end{array}$} & \multicolumn{2}{|c|}{$\begin{array}{l}8-0 \mathrm{HdG} \\
\text { negative } \\
(n=27)\end{array}$} & \multicolumn{2}{|c|}{$\begin{array}{l}8-0 \mathrm{HdG} \\
\text { positive } \\
(n=31)\end{array}$} & \multirow{2}{*}{$\begin{array}{l}P \\
\text { value* }\end{array}$} & \multicolumn{2}{|c|}{$\begin{array}{l}8-0 \mathrm{HdG} \\
\text { UNK } \\
(n=14) \\
\end{array}$} & \multicolumn{2}{|c|}{$\begin{array}{l}8-0 \mathrm{HdG} \\
\text { negative } \\
(n=22) \\
\end{array}$} & \multicolumn{2}{|c|}{$\begin{array}{l}8-0 \mathrm{HdG} \\
\text { positive } \\
(n=24) \\
\end{array}$} & \multirow{2}{*}{$\begin{array}{l}P \\
\text { value* }^{*}\end{array}$} \\
\hline & $n$ & $\%$ & $n$ & $\%$ & $n$ & $\%$ & & $n$ & $\%$ & $n$ & $\%$ & $n$ & $\%$ & \\
\hline $\begin{array}{l}\text { Age, mean (range), } \\
\text { years }\end{array}$ & - & - & 63 & $(37-82)$ & 61 & $(42-77)$ & 0.415 & - & - & 63 & $(46-83)$ & 59 & $(43-78)$ & 0.110 \\
\hline \multicolumn{15}{|l|}{ Sex } \\
\hline Male & - & - & 20 & 74 & 22 & 71 & 0.792 & - & - & 14 & 64 & 14 & 58 & 0.713 \\
\hline Female & - & - & 7 & 26 & 9 & 29 & & - & - & 8 & 36 & 10 & 42 & \\
\hline \multicolumn{15}{|l|}{ Onset } \\
\hline Synchronous & - & - & 14 & 52 & 22 & 71 & 0.134 & - & - & 12 & 55 & 10 & 42 & 0.382 \\
\hline Metachronous & - & - & 13 & 48 & 9 & 29 & & - & - & 10 & 46 & 14 & 58 & \\
\hline \multicolumn{15}{|l|}{ Treatment status } \\
\hline Treated & - & - & 6 & 22 & 9 & 29 & 0.555 & - & - & 0 & 0 & 9 & 38 & 0.001 \\
\hline Untreated & - & - & 21 & 78 & 22 & 71 & & - & - & 22 & 100 & 15 & 63 & \\
\hline
\end{tabular}

${ }^{*} \mathrm{All} \chi^{2}$ test (categorical data), except for the continuous variable age at surgery (independent-sample $t$-test).

8-OHdG, 8-hydroxy-2'-deoxyguanosine; UNK, unknown.

\section{Accumulation of Oxidative Damage in Therapy-Exposed Tumors}

Chemotherapy and radiotherapy generate oxidative damage. ${ }^{11,12,23-25}$ Therefore, we hypothesized that the increased accumulation of oxidative DNA damage in CRC liver metastases could be a consequence of differences in treatment status between primary CRC tumors and their corresponding liver metastases. To test this hypothesis, tissues were stratified according to their treatment status, and expression of the different markers was reanalyzed. Treatment before tumor resection was associated with a significant increase of $\gamma \mathrm{H} 2 \mathrm{AX}$ levels, both in primary tumors and metastases $(P \leq 0.05)$ (Figure $2 \mathrm{~A})$. In addition, $\gamma \mathrm{H} 2 \mathrm{AX}$ levels were higher in previously untreated metastases compared with untreated primary tumors (Figure 2A).

Levels of 8-OHdG were increased in therapy-exposed metastases $(P \leq 0.01)$ (Figure 2B), and a similar trend was observed in primary tumors (Figure 2B). Similar to $\gamma \mathrm{H} 2 \mathrm{AX}$, 8-OHdG levels were also increased in previously untreated liver metastases when compared with untreated primary tumors (Figure 2B).

\section{Accumulation of Oxidative Damage in Therapy-Exposed Mesenchymal-Like Tumors}

The recent classification of colorectal tumors into molecular subtypes $^{13}$ offers a new paradigm for developing subtypetargeted therapy. One of the subtypes, CMS4, is characterized by a mesenchymal gene expression profile and is associated with a poor prognosis ${ }^{13}$ and high expression of hydrogen peroxide-regulated genes (Supplemental Figure S2). ${ }^{9}$ To assess whether high expression of hydrogen peroxide-regulated genes in mesenchymal-like tumors is associated with increased oxidative damage, immunohistochemistry-based molecular classification was used to discriminate between EPI- and MES-like tumors. ${ }^{18}$ Previously untreated primary tumors and metastases of the MES-like subtype showed significantly less accumulation of $\gamma \mathrm{H} 2 \mathrm{AX}$, and there was a trend toward less accumulation of 8 -OHdG $(P=0.054$ for 8 -OHdG primary; $P=0.057$ for 8-OHdG metastasis) (Figure 3, A and B). Of interest, levels of $\gamma \mathrm{H} 2 \mathrm{AX}$ and $8-\mathrm{OHdG}$ lesions were significantly higher in MES-like tumors (primary and metastases) that received prior treatment, but not in EPI-like tumors (Figure 3, A and B).

\section{Low Levels of Phosphorylated NRF2 and Its Target Genes in Mesenchymal-Like Tumors}

NRF2 is a transcription factor that induces expression of many antioxidant genes in normal and cancer cells and is considered to be (one of) the most important orchestrators of the cellular antioxidant response. ${ }^{14}$ Therefore, it was tested whether the levels of active (phosphorylated S40) NRF2 were different between primary tumors and metastases, and between MES- and EPI-like tumors. Primary tumors and liver metastases contained similar levels of phosphorylated NRF2 (Figure 4A). Furthermore, a highly significant negative correlation was found between phosphorylated NRF2 levels and an MES-like tumor phenotype (Figure 4B and Table 1). Analysis of NRF2 target genes (ie, genes containing NRF2-binding sites, as demonstrated by chromatin immunoprecipitation sequencing ${ }^{26}$ ) in a large composite CRC cohort showed that MES-like tumors (CMS4) express NRF2 target genes-as a proxy for active NRF2-at significantly lower levels than epithelial subtypes (CMS2 and CMS3) (Figure 4C and Supplemental Figure S3). In addition, low NRF2 mRNA levels in liver metastases were also associated with poor OS, and this effect was most prominent in MES-like tumors (Figure 4D). 


\section{8-OHdG Staining in Mesenchymal-Like Metastases Is Associated with Poor Survival}

The abundance of oxidative damage in resected tumors may not only serve as an indicator of prior treatment but could also be related to protumorigenic oxidative stress signaling. Therefore, OS of patients with EPI- and MESlike metastases with positive (H-score $>0)$ and negative $(\mathrm{H}$-score $=0)$ 8-OHdG staining was analyzed. A positive 8 -OHdG status $(n=23)$ in MES-like metastases showed a trend toward significance $(P=0.119)$ as a marker for poor OS (Figure 5A). In contrast, a positive $8-\mathrm{OHdG}$ status in EPI-like metastases was not associated with survival (Figure 5B). In addition, a positive treatment status was associated with a positive $8-\mathrm{OHdG}$ status in MES-like metastases but not in EPI-like metastases (Table 2).

\section{Discussion}

CRC patients with metastatic disease have a poor prognosis, ${ }^{3,4}$ and effective therapeutic approaches that have an impact on long-term survival are urgently needed. Targeting the increased redox status of tumor cells could be such an approach. These data show that CRC liver metastases are characterized by high levels of oxidative DNA damage. This is in line with a recent report showing that metastatic melanoma nodules express significantly higher levels of ROS in a human melanoma xenograft model.${ }^{10}$ Of interest, these data point to higher oxidative stress levels in treatment-naïve CRC metastases compared with primary tumors. This indicates that factors distinct from treatment status, such as microenvironmental cues and/or intrinsic differences between metastasis-competent and metastasisincompetent tumor cell clones, could be responsible for the different levels of oxidative damage in primary tumors and metastases.

Expression of the DNA damage markers $\gamma \mathrm{H} 2 \mathrm{AX}$ and 8-OHdG in CRC liver metastases was significantly higher in patients who had received treatment. This may be because of a sustained chemotherapy- and radiotherapy-induced elevation of $\operatorname{ROS}^{12,23}$ and $8-\mathrm{OHdG}$ lesions. ${ }^{24}$ Additional experiments will be necessary to assess the causal relationship between treatment and redox status. Such studies will require the prospective collection of tumor biopsy specimens before and after treatment.

Effective therapies for poor-prognosis MES-like CRC tumors $^{13,15,18}$ are urgently needed. These tumors were found to be characterized by low expression of $\gamma \mathrm{H} 2 \mathrm{AX}, 8-\mathrm{OHdG}$, and phosphorylated NRF2. Although phosphorylated NRF2 might not adequately reflect NRF2 activation status, ${ }^{27}$ MESlike tumors also expressed low levels of NRF2 target genes. Moreover, low mRNA levels of NFE2L2 (the gene encoding NRF2) in primary tumors were associated with poor survival in the colorectal adenocarcinoma cohort, as shown by the Human Protein Atlas (https://www.proteinatlas.org/ ENSG00000116044-NFE2L2/pathology/tissue/colorectal+ cancer, last accessed October 31, 2017). Finally, low levels of NRF2 mRNA in CRC metastases identified a subgroup of MES-like tumors with a poor OS.

The correlation of low phosphorylated NRF2 protein levels, low NFE2L2 mRNA, and low NRF2 target gene expression with an aggressive tumor phenotype is somewhat surprising, considering that sustained and constitutive activation of NRF2 is known to promote tumor growth ${ }^{28}$ and chemotherapy resistance. ${ }^{29}$ Possibly, mRNA or phosphorylated NRF2 levels may not adequately reflect total NRF2 activity. Alternatively, NRF2 regulation of cancer cell viability may be context dependent. Finally, the levels of NRF2 expressed at the time of tumor resection may not reflect the ability of these tumors to mount an NRF2 response and increase their antioxidant capacity in response to ROS insults. Indeed, phosphorylated NRF2 protein levels were significantly higher in MES-like tumors that had been exposed to prior treatment. Although markers of oxidative damage were also higher in these tumors, they clearly survived treatment. This suggests that MES-like tumors may have a higher tolerance for therapy-induced oxidative damage than EPI-like tumors, which may (partly) explain their relative resistance to chemotherapy. ${ }^{30}$ Of importance, a definitive assessment of the role of NRF2, its phosphorylation, and activation of its target genes in colorectal cancer progression requires further studies in model systems and human tissues. In particular, it will be interesting to investigate whether a higher tolerance for oxidative stress, possibly relying on NRF2 activity, contributes to the higher metastatic potential of aggressive MES-like tumors.

Given that moderately increased levels of oxidative stress may have tumor-promoting effects, ${ }^{7}$ it was explored whether a positive 8 -OHdG status identifies aggressive CRC metastases. Of interest, these data suggest that patients with 8-OHdG-positive MES-like metastases have a shorter OS, whereas 8-OHdG status did not seem to be associated with $\mathrm{OS}$ in patients with EPI-like tumors. These results, therefore, suggest that a further subclassification (based on redox status) may improve the prognostic value of colon cancer subtyping, at least in the metastatic setting. Finally, this may help selecting patients for specific types of targeted therapies, particularly those targeting the redox status of tumor cells. The value of such an approach should now be evaluated in preclinical studies using models of MES- and EPI-like CRC.

In conclusion, therapeutics that interfere with redox status might be particularly effective in targeting metastases, therapy-surviving tumor cells, and MES-like tumors and might be administered in combination with conventional oxidative stress-inducing radiotherapeutics and chemotherapeutics. Further studies will be needed to clarify whether markers of redox status could have prognostic and/ or predictive value. 


\section{Supplemental Data}

Supplemental material for this article can be found at https://doi.org/10.1016/j.ajpath.2018.06.008.

\section{References}

1. Ferlay J, Steliarova-Foucher E, Lortet-Tieulent J, Rosso S, Coebergh JW, Comber H, Forman D, Bray F: Cancer incidence and mortality patterns in Europe: estimates for 40 countries in 2012. Eur J Cancer 2013, 49:1374-1403

2. Siegel R, Desantis C, Jemal A: Colorectal cancer statistics, 2014. CA Cancer J Clin 2014, 64:104-117

3. Loupakis F, Cremolini C, Masi G, Lonardi S, Zagonel V, Salvatore L, Cortesi E, Tomasello G, Ronzoni M, Spadi R, Zaniboni A, Tonini G, Buonadonna A, Amoroso D, Chiara S, Carlomagno C, Boni C, Allegrini G, Boni L, Falcone A: Initial therapy with FOLFOXIRI and bevacizumab for metastatic colorectal cancer. N Engl J Med 2014, 371:1609-1618

4. Cremolini C, Schirripa M, Antoniotti C, Moretto R, Salvatore L, Masi G, Falcone A, Loupakis F: First-line chemotherapy for mCRC: a review and evidence-based algorithm. Nat Rev Clin Oncol 2015, 12: 607-619

5. Trachootham D, Zhou Y, Zhang H, Demizu Y, Chen Z, Pelicano H, Chiao PJ, Achanta G, Arlinghaus RB, Liu J, Huang P: Selective killing of oncogenically transformed cells through a ROS-mediated mechanism by beta-phenylethyl isothiocyanate. Cancer Cell 2006, 10: 241-252

6. Cairns RA, Harris IS, Mak TW: Regulation of cancer cell metabolism. Nat Rev Cancer 2011, 11:85-95

7. Wiseman H, Halliwell B: Damage to DNA by reactive oxygen and nitrogen species: role in inflammatory disease and progression to cancer. Biochem J 1996, 313(Pt 1):17-29

8. Schafer ZT, Grassian AR, Song L, Jiang Z, Gerhart-Hines Z, Irie HY, Gao S, Puigserver P, Brugge JS: Antioxidant and oncogene rescue of metabolic defects caused by loss of matrix attachment. Nature 2009, 461:109-113

9. Emmink BL, Laoukili J, Kipp AP, Koster J, Govaert KM, Fatrai S, Verheem A, Steller EJ, Brigelius-Flohe R, Jimenez CR, Borel Rinkes IH, Kranenburg O: GPx2 suppression of $\mathrm{H} 2 \mathrm{O} 2$ stress links the formation of differentiated tumor mass to metastatic capacity in colorectal cancer. Cancer Res 2014, 74:6717-6730

10. Piskounova E, Agathocleous M, Murphy MM, Hu Z, Huddlestun SE, Zhao Z, Leitch AM, Johnson TM, DeBerardinis RJ, Morrison SJ: Oxidative stress inhibits distant metastasis by human melanoma cells. Nature 2015, 527:186-191

11. Alexandre J, Nicco C, Chereau C, Laurent A, Weill B, Goldwasser F, Batteux F: Improvement of the therapeutic index of anticancer drugs by the superoxide dismutase mimic mangafodipir. J Natl Cancer Inst 2006, 98:236-244

12. Arifa RD, Madeira MF, de Paula TP, Lima RL, Tavares LD, MenezesGarcia Z, Fagundes CT, Rachid MA, Ryffel B, Zamboni DS, Teixeira MM, Souza DG: Inflammasome activation is reactive oxygen species dependent and mediates irinotecan-induced mucositis through IL-1beta and IL-18 in mice. Am J Pathol 2014, 184:2023-2034

13. Guinney J, Dienstmann R, Wang X, de Reynies A, Schlicker A, Soneson C, et al: The consensus molecular subtypes of colorectal cancer. Nat Med 2015, 21:1350-1356

14. Gorrini C, Harris IS, Mak TW: Modulation of oxidative stress as an anticancer strategy. Nat Rev Drug Discov 2013, 12:931-947
15. Trumpi K, Ubink I, Trinh A, Djafarihamedani M, Jongen JM, Govaert KM, Elias SG, van Hooff SR, Medema JP, Lacle MM, Vermeulen L, Borel Rinkes IHM, Kranenburg O: Neoadjuvant chemotherapy affects molecular classification of colorectal tumors. Oncogenesis 2017, 6:e357

16. van Diest PJ: No consent should be needed for using leftover body material for scientific purposes. BMJ 2002, 325:648-651

17. Coebergh JWW on behalf of the FMWV Society: Federa: Human Tissue and Medical Research: Code of Conduct for Responsible Use (2011). Rotterdam, the Netherlands: FMWV, 2011

18. Trinh A, Trumpi K, De Sousa EMF, Wang X, de Jong JH, Fessler E, Kuppen PJ, Reimers MS, Swets M, Koopman M, Nagtegaal ID, Jansen M, Hooijer GK, Offerhaus GJ, Kranenburg O, Punt CJ, Medema JP, Markowetz F, Vermeulen L: Practical and robust identification of molecular subtypes in colorectal cancer by immunohistochemistry. Clin Cancer Res 2017, 23:387-398

19. van Diest PJ, van Dam P, Henzen-Logmans SC, Berns E, van der Burg ME, Green J, Vergote I; European Organization for Research and Treatment of Cancer-Gynaecological Cancer Cooperative Group: A scoring system for immunohistochemical staining: consensus report of the task force for basic research of the EORTC-GCCG. J Clin Pathol 1997, 50:801-804

20. Snoeren N, van Hooff SR, Adam R, van Hillegersberg R, Voest EE, Guettier C, van Diest PJ, Nijkamp MW, Brok MO, van Leenen D, Koerkamp MJ, Holstege FC, Rinkes IH: Exploring gene expression signatures for predicting disease free survival after resection of colorectal cancer liver metastases. PLoS One 2012, 7:e49442

21. Kuo LJ, Yang LX: Gamma-H2AX: a novel biomarker for DNA double-strand breaks. In Vivo 2008, 22:305-309

22. Klaunig JE, Kamendulis LM, Hocevar BA: Oxidative stress and oxidative damage in carcinogenesis. Toxicol Pathol 2010, 38:96-109

23. Tulard A, Hoffschir F, de Boisferon FH, Luccioni C, Bravard A: Persistent oxidative stress after ionizing radiation is involved in inherited radiosensitivity. Free Radic Biol Med 2003, 35:68-77

24. Siomek A, Tujakowski J, Gackowski D, Rozalski R, Foksinski M, Dziaman T, Roszkowski K, Olinski R: Severe oxidatively damaged DNA after cisplatin treatment of cancer patients. Int J Cancer 2006, 119:2228-2230

25. Preston TJ, Henderson JT, McCallum GP, Wells PG: Base excision repair of reactive oxygen species-initiated 7,8-dihydro-8-oxo-2'deoxyguanosine inhibits the cytotoxicity of platinum anticancer drugs. Mol Cancer Ther 2009, 8:2015-2026

26. Hirotsu Y, Katsuoka F, Funayama R, Nagashima T, Nishida Y, Nakayama K, Engel JD, Yamamoto M: Nrf2-MafG heterodimers contribute globally to antioxidant and metabolic networks. Nucleic Acids Res 2012, 40:10228-10239

27. Sun Z, Huang Z, Zhang DD: Phosphorylation of Nrf2 at multiple sites by MAP kinases has a limited contribution in modulating the Nrf2-dependent antioxidant response. PLoS One 2009, 4:e6588

28. Ma Q: Role of nrf2 in oxidative stress and toxicity. Annu Rev Pharmacol Toxicol 2013, 53:401-426

29. Wang XJ, Sun Z, Villeneuve NF, Zhang S, Zhao F, Li Y, Chen W, Yi X, Zheng W, Wondrak GT, Wong PK, Zhang DD: Nrf2 enhances resistance of cancer cells to chemotherapeutic drugs, the dark side of Nrf2. Carcinogenesis 2008, 29:1235-1243

30. Song N, Pogue-Geile KL, Gavin PG, Yothers G, Kim SR, Johnson NL, Lipchik C, Allegra CJ, Petrelli NJ, O'Connell MJ, Wolmark N, Paik S: Clinical outcome from oxaliplatin treatment in stage II/III colon cancer according to intrinsic subtypes: secondary analysis of NSABP C-07/NRG oncology randomized clinical trial. JAMA Oncol 2016, 2:1162-1169 